\title{
Subcutaneous Interleukin-2 Monotherapy for Metastatic Renal Cell Carcinoma in Korean Patients
}

\author{
Jeong Ho Kim¹, Ki Soo Lee ${ }^{2}$, Choung-Soo Kim³, Young Deuk Choi ${ }^{4}$, Tae Hyo Kim² \\ 'Department of Urology, Inje University Haeundae Paik Hospital, Inje University College of Medicine, Busan, Korea \\ ${ }^{2}$ Department of Urology, Dong-A University College of Medicine, Busan, Korea \\ ${ }^{3}$ Department of Urology, Asan Medical Center, University of Ulsan College of Medicine, Seoul, Korea \\ ${ }^{4}$ Department of Urology, Severance Hospital, Urological Science Institute, Yonsei University College of Medicine, Seoul, \\ Korea
}

Purpose: This study was a prospective single-arm clinical trial aimed at assessing the efficacy and toxicity of subcutaneous interleukin (IL)-2 monotherapy in patients with metastatic renal cell carcinoma (RCC).

Materials and Methods: We enrolled 26 patients with metastatic RCC in this multicenter controlled trial. The patients received subcutaneous injections of recombinant IL-2 (BMI-rh-IL2, an aldesleukin biosimilar, BMIKOREA Co., Ltd.) in 5-week cycles. In the first week, the patients received a subcutaneous IL-2 loading dose of $18 \times 10^{6} \mathrm{IU}$ once on treatment days $1-5$, followed by 2 days of rest. In the following 3 weeks, they received a dose of $18 \times 10^{6} \mathrm{IU}$ via subcutaneous injection once on treatment days 1 and 2 . Then, the patients received a dose of $9 \times 10^{6} \mathrm{IU}$ via subcutaneous injection once on treatment days 3,4, and 5, followed by 2 days of rest. The primary end point was the objective response rate; the secondary end points were progressionfree survival (PFS) and safety.

Results: Overall, 22 patients were included in the final per-protocol analysis. The objective response and the disease control rates were $13.64 \%$ (3 of 22), and $90.9 \%$ (20 of 22), respectively. The mean PFS was 5.55 months (95\% confidence interval, 2.71-8.4). The proportion of patients who experienced a treatment-related grade 3 or 4 adverse event was $3.85 \%$ (1 of 26). There were no treatment-related deaths.

Conclusions: In this study, the subcutaneous IL-2 monotherapy regimen demonstrated efficacy and safety comparable to those reported in previous studies of subcutaneous IL-2 monotherapy and was effective in Korean patients with metastatic RCC.

Key Words: Renal cell carcinoma, Interleukin-2, Clinical trial, Metastasis, Subcutaneous injections
Received August 11, 2021

Revised September 14, 2021

Accepted September 17, 2021

Corresponding author:

Tae Hyo Kim

Email: dockim0927@hanmail.net

https://orcid.org/0000-0002-5994-7878

\section{INTRODUCTION}

In recent years, the instances of incidental detection of early and small renal tumors, which are potentially curable, are increasing rapidly because of an increase in regular health checkups worldwide. In most patients, local or locally advanced renal cell carcinoma (RCC) is curable; however, some of these patients develop metastatic disease. In addition, many patients are still diagnosed with metastatic RCC. ${ }^{1}$ The prognosis of metastatic RCC, as in the case of most metastatic cancers in humans, is generally considered poor, with the predicted 5-year survival rate being lower 
than $20 \% .^{2-4}$ Owing to a deeper understanding of the molecular biology of RCC, the therapeutic options for metastatic RCC have expanded, and several antiangiogenic agents and immunecheckpoint inhibitors (ICIs) have been developed. Tyrosine kinase inhibitors (TKIs), especially sunitinib and pazopanib, are currently used as the first-line treatments for metastatic RCC and have shown promising results., ${ }^{5,6}$ Satisfactory results have also been reported for the more recently developed ICIs. ${ }^{7,8}$ However, several issues remain to be addressed. Moreover, further study of the optimal treatment sequences for patients with metastatic RCC is needed.

RCC is resistant to cytotoxic chemotherapy. The mechanisms underlying the development of cytotoxic drug resistance in metastatic RCC remain unknown. It is generally accepted that these mechanisms may involve overexpression of p-glycoprotein efflux pumps and the dysregulation of the microtubule-hypoxia inducible factor signaling axis. ${ }^{9}$ Although some clinical outcomes of cytotoxic chemotherapy in nonclear cell RCC, such as sarcomatoid and collecting-duct RCC variants, have been reported, the roles of cytotoxic chemotherapy in the treatment of clear cell RCC are poorly elucidated. ${ }^{9-12}$ Thus, other effective therapeutic strategies, such as immune modulation, have been intensively studied. Cytokine therapies have been used for the treatment of metastatic RCC for a long time, and antiangiogenic therapies were introduced in 2005. At present, ICIs that regulate $\mathrm{T}$-cell activation are widely used.

The role of cytokines and ICIs in the cancer immunotherapy is to stimulate the immune response through several different mechanisms. In particular, cytokines are used to non-specifically stimulate immune processes. ${ }^{13}$ To date, 2 cytokinesinterleukin-2 (IL-2) and interferon- $\alpha$-have been approved for use as single agents for the treatment of several cancers. These agents have demonstrated significant antitumor activity against advanced RCC and metastatic melanoma. ${ }^{14}$ High-dose (HD) IL-2 therapy was approved in 1992 for the treatment of metastatic RCC; most of the endogenous IL-2 is produced by activated CD4+ T cells. Administered IL-2 increases natural killer (NK) cell activity and induces lymphokine-activated killer cells in the circulation. ${ }^{15}$ These cytotoxic effector cells lead to the eradication of tumor cells. In a phase II study in which HD IL-2 therapy was performed in 255 patients with metastatic RCC, the overall objective response rate (ORR) was $14 \%$, with the complete response (CR) and partial response (PR) rates being $5 \%$ and $9 \%$, respectively. ${ }^{16}$ However, HD IL-2 therapy is associated with a higher incidence of adverse events (AEs), which can be life-threatening when improperly managed. It is also associated with a severe toxicity profile, and therefore, lowdose and subcutaneous regimens have been suggested. In this prospective single-arm controlled study of IL-2, we evaluated its efficacy and safety in Korean patients with metastatic RCC.

\section{MATERIALS AND METHODS}

\section{Patients and Ethical Statement}

We included patients with clinically diagnosed metastatic RCC who received subcutaneous IL-2 therapy between December 2011 and November 2014 at 3 tertiary care centers (Asan Medical Center, Dong-A University Hospital, Severance Hospital). The major eligibility criteria were as follows; age of $\geq 18$ to $\leq 75$ years; an Eastern Cooperative Oncologic Group (ECOG) performance status of 0 or 1 ; a histologically or cytologically confirmed diagnosis of metastatic RCC with a bidimensionally measurable and clearly progressive lesion; life expectancy of $\geq$ 10 weeks; favorable or intermediate Memorial Sloan Kettering Cancer Center (MSKCC) risk; 
and an adequate organ function. Adequate organ function was defined as follows: normal hematologic parameters, a serum creatinine level of $\leq$ twice the upper limit of normal (ULN); serum glutamic oxaloacetate transaminase and glutamate pyruvate transaminase levels $\leq$ twice the ULN; and a serum bilirubin level of $\leq 2.0 \mathrm{mg} /$ dL. Patients were able to receive pressor agents. The exclusion criteria were as follows: a history of hypersensitive reaction due to recombinant IL-2, other malignancies, more than 12 metastatic sites, a history or symptoms of severe cardiac disease, systemic infections requiring antibiotic treatment, metastasis to the central nervous system, resting $\mathrm{pO}_{2}$ of $<60 \mathrm{mmHg}$, presence of organ allografts, corticosteroid dependence, presence of immunodeficiency disorder, and pregnancy or lactation.

The study was approved by the Institutional Review Board of all the participating institutions (Asan Medical Center: 2010-0883, Dong-A University Hospital: 11-117, Severance Hospital: 4-2011-0038) and conducted according to the ethical standards laid down by the 1964 Declaration of Helsinki and its later amendments. Voluntary written informed consent was obtained from all study participants.

\section{Treatment Protocol}

BMI-recombinant IL-2 (BMI-rh-IL-2), a biosimilar of aldesleukin (Proleukin; Chiron Italia s.s.l, Milan, Italy) with an identical molecular structure, was used in this study. In the first week, on treatment days $1-5$, patients received a subcutaneous recombinant human IL-2 (BMI-rhIL2, BMIKOREA CO., LTD., Jeju, Korea) loading dose of $18 \times 10^{6}$ IU once followed by 2 days of rest. In the following 3 weeks, on treatment days 1 and 2 , patients received a dose of $18 \times 10^{6}$ IU via subcutaneous injection at once. Then, on treat- ment days 3, 4, and 5, the patients received a dose of $9 \times 10^{6} \mathrm{IU}$ via subcutaneous injection at once, followed by 2 days of rest. After treatment for 4 weeks, the patients were given a 1 -week rest. The cycles were repeated every 5 weeks. One cycle consisted of 4 weeks of treatment followed 1 week of rest. The treatment schedule was based on those in previous study. ${ }^{17}$ Treatment was continued until progressive disease or unacceptable toxicity was encountered. If significant toxicity occurred during treatment, a treatment delay of up to 4 weeks was allowed for the resolution of side effects between courses. Treatment delay was defined as not receiving the subsequent treatment within a week of the previous treatment.

\section{Response and Toxicity Assessment}

Patients were evaluated for response and progression each cycle after the end of the treatment cycle for study termination. The criteria for CR and PR assessment were based on Response Evaluation Criteria in Solid Tumors (ver. 1.1) guidelines. The primary and secondary end points were ORR and progression-free survival (PFS) time, respectively. Treatment safety was assessed according to the Common Terminology Criteria for Adverse Events version 3.0. Patient data such as age, sex, ECOG performance status, histologic subtype, prior therapy, location of metastatic sites, laboratory findings, and PFS were prospectively collected over a 1-year period according to the study plan.

\section{Statistical Analysis}

In the planned analysis, efficacy was evaluated in the per-protocol (PP) population, and the intent-to-treat (ITT) population was mainly used to assess the safety outcome. Categorical variables are expressed as frequencies and percentages. 
Continuous variables are expressed as the mean \pm standard deviation or median (interquartile range) values. PFS was defined as the period from the date of the first IL-2 dose to the date of progression. All survival outcomes were analyzed using the Kaplan-Meier analysis. All statistical analyses were performed using IBM SPSS Statistics ver. 20.0 (IBM Co., Armonk, NY, USA), with a 2 -sided $\mathrm{p}$-value of $<0.05$ indicating statistical significance.

\section{RESULTS}

Between December 2011 and November 2014, 36 patients were screened at the 3 participating institutions. Of these 36 patients, 26 who met the inclusion criteria were enrolled in the trial (ITT population), and 22 patients finally followed the clinical trial protocol (PP population) (Fig. 1). Four patients were dropped from the final analysis because a follow-up test could not be performed. The clinical characteristics of the 26 patients are listed in Table 1. Fourteen patients (53.9\%) had previously received target or immunologic agents, and 1 patient had received radiation therapy for pain control within 4 weeks of the screening. All patients were in the favorable or intermediate risk groups according to the MSKCC prognostic criteria. The mean time from the diagnosis of metastatic RCC to IL-2 administration was $20.16 \pm$ 23.64 months.

Among the 22 patients (PP population), none

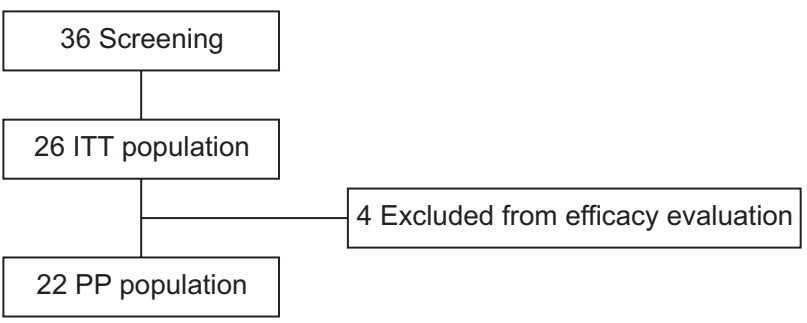

Fig. 1. Flow chart showing study subject enrollment. ITT: intention-to-treat, PP: per-protocol. achieved CR, 3 (13.6\%) achieved PR, and 17 (77.3\%) achieved stable disease (Table 2). The ORR and disease control rate (DCR) were $13.6 \%$ and 90.9\%, respectively (Table 2). The mean PFS was 5.55 months (95\% confidence interval, 2.71-8.4) (Fig. 2 ). Among the 26 patients (ITT population), the overall incidence rates of AEs and adverse drug reactions (ADRs) were $84.6 \%(n=22)$ and $76.9 \%$ $(n=20)$, respectively. The incidence rates of severe AEs and ADRs were 19.2\% $(n=5)$ and 3.9\% $(n=1)$, respectively (Table 3 ). The most common ADRs were rigors/chills $(n=10)$, and fever $(n=8)$. The

Table 1. Patients' baseline characteristics

\begin{tabular}{|c|c|c|}
\hline Characteristic & ITT $(\mathrm{N}=26)$ & $\mathrm{PP}(\mathrm{N}=22)$ \\
\hline Age (yr), mean \pm SD & $59.9 \pm 8.6$ & $59.9 \pm 8.7$ \\
\hline \multicolumn{3}{|l|}{ Sex } \\
\hline Male & $21(80.8)$ & $17(77.3)$ \\
\hline Female & $5(19.2)$ & $5(22.7)$ \\
\hline Body weight (kg), median (IQR) & $68.2(60.3-76.7)$ & $68.4(61.7-76.7)$ \\
\hline Diabetes mellitus & $6(23.1)$ & $5(22.7)$ \\
\hline Hypertension & $7(26.9)$ & $6(27.3)$ \\
\hline \multicolumn{3}{|l|}{ ECOG performance status } \\
\hline 0 & $19(73.1)$ & $17(77.3)$ \\
\hline 1 & $7(26.9)$ & $5(22.7)$ \\
\hline \multicolumn{3}{|l|}{ Histopathologic finding } \\
\hline Clear cell & $22(84.6)$ & $19(86.4)$ \\
\hline Nonclear cell & $4(15.4)$ & $3(13.6)$ \\
\hline \multicolumn{3}{|l|}{ Metastatic sit (n) } \\
\hline Lung & $18(69.2)$ & $15(68.2)$ \\
\hline Bone & $6(23.1)$ & $5(22.7)$ \\
\hline Liver & $4(15.4)$ & $4(18.2)$ \\
\hline Lymph node & $7(26.9)$ & $6(27.3)$ \\
\hline Others & $8(30.8)$ & $0(0)$ \\
\hline \multicolumn{3}{|l|}{ Treatment setting } \\
\hline First line & $12(46.2)$ & $10(45.5)$ \\
\hline Second line & $5(19.2)$ & $4(18.2)$ \\
\hline Third line or higher & $9(34.6)$ & $8(36.4)$ \\
\hline Prior treatment & $14(53.9)$ & $12(54.6)$ \\
\hline Target agent & $14(100)$ & $12(100)$ \\
\hline Sunitinib & $8(57.1)$ & $7(58.3)$ \\
\hline Sorafenib & $7(50)$ & $7(58.3)$ \\
\hline Axitinib & $1(7.1)$ & $0(0)$ \\
\hline Everolimus & $8(57.1)$ & $7(58.3)$ \\
\hline Interferons & $1(7.1)$ & $1(8.3)$ \\
\hline Interleukins & $1(7.1)$ & $1(8.3)$ \\
\hline Previous radiotherapy & $1(3.8)$ & $1(4.5)$ \\
\hline
\end{tabular}

Values are presented as number (\%) unless otherwise indicated. ITT: intention-to-treat, PP: per-protocol, SD: standard deviation, IQR: interquartile range, ECOG: Eastern Cooperative Oncology Group Performance Status. 
Table 2. Clinical outcomes

\begin{tabular}{lcc}
\hline \multicolumn{1}{c}{ Variable } & ITT & PP \\
\hline $\mathrm{CR}$ & $0(0)$ & $0(0)$ \\
$\mathrm{PR}$ & $3(11.5)$ & $3(13.6)$ \\
$\mathrm{SD}$ & $21(80.8)$ & $17(77.3)$ \\
$\mathrm{PD}$ & $2(7.7)$ & $2(9.1)$ \\
Objective response rate & $3(11.5)$ & $3(13.6)$ \\
Disease control rate & $24(92.3)$ & $20(90.9)$ \\
Total & $26(100)$ & $22(100)$ \\
Progression-free survival & $6.7(3.36-10.02)$ & $5.55(2.71-8.4)$ \\
$\quad($ mo $)(95 \% \mathrm{CI})$ & & \\
\hline
\end{tabular}

Values are presented as number (\%) unless otherwise indicated. ITT: intention-to-treat, PP: per-protocol, CR: complete response, PR: partial response, SD: stable disease, $\mathrm{PD}$ : progressive disease, $\mathrm{CI}$ : confidence interval.

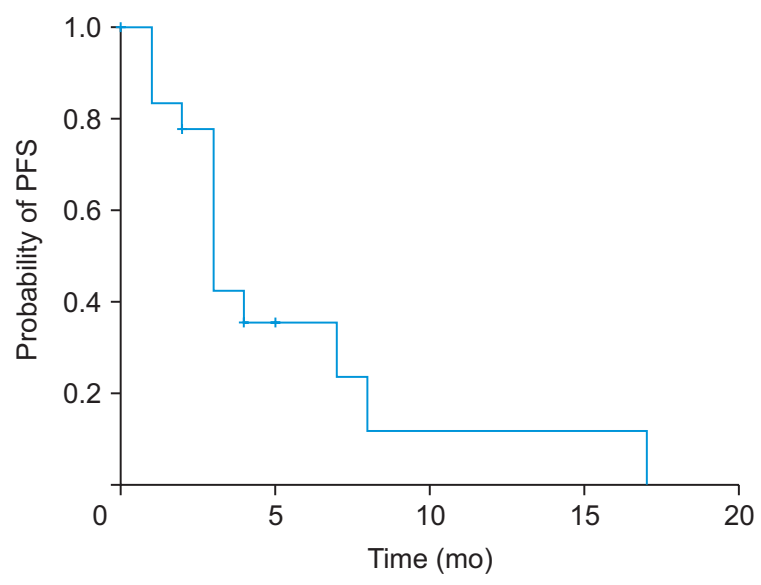

No. at risk 21
1

1

Fig. 2. Kaplan-Meier survival curve of progression-free survival (PFS) in the per-protocol population.

most common laboratory test abnormality was increased serum alanine aminotransferase level $(n=4)$. The most severe ADR was general weakness $(n=1)$, and the affected patient made a complete recovery with hospital supportive care. Treatment delay was not attributable to AEs or ADRs in all patients.

\section{DISCUSSION}

Several studies on IL-2 have provided clinicians and patients with meaningful outcomes. However, no prospective randomized controlled study has

Table 3. Adverse drug reaction grade 3 or 4 (ITT population)

\begin{tabular}{lccc}
\hline \multicolumn{1}{c}{ Variable } & No. of patients & Grade & Treatment relationships \\
\hline Anemia & 1 & 3 & No \\
Ascites & 1 & 3 & No \\
Melena & 1 & 3 & No \\
Cerebral infarction & 1 & 3 & No \\
Pain - other & 1 & 3 & No \\
General weakness & 1 & 4 & Yes \\
\hline
\end{tabular}

ITT: intention-to-treat.

examined the clinical outcomes of IL-2 therapy in Asian patients. This prospective single-arm trial was conducted to evaluate the efficacy and safety of subcutaneous IL-2 monotherapy for the treatment of metastatic RCC in Koreans. To this end, the enrolled patients were administered IL-2 regardless of prior therapy. Previous studies of subcutaneous IL-2 monotherapy reported an ORR of $10 \%$ to $23 \% .{ }^{17-19}$ We found that the response rate was comparable to those reported previously (13.6\% vs. $20 \%$; $\mathrm{p}=0.344) .{ }^{17-19}$

In recent studies, the ORR with HDIL-2 treatment has been reported as high as $35 \%{ }^{3,4}$ It is difficult to explain the difference in ORR between our study and recent ones; however, the difference may be attributable to several factors such as the method of treatment administration, the dosage of treatment, and patient characteristics. In addition, the mean time to study participation from the time of diagnosis of metastatic disease was too long, which influenced the survival outcomes. Furthermore, the DCR in our study was $90.5 \%$ and consistent with the DCR of $92 \%$ in another study (90.5\% vs. 92\%; p=0.109). ${ }^{3}$ We believe that DCR may be a more practical parameter for evaluating treatment efficacy. ${ }^{20}$

IL-2 has been administered in several different ways for a long time. In the early days, patients treated with HD IL-2 experienced severe AEs such as hypotension, infection, and thrombocytopenia, and often required intensive care. ${ }^{16,21}$ Hence HD IL-2 has not been widely used, and it is not commonly used today as first-line therapy for 
metastatic RCC. Initial reports from the latest studies suggest that HD IL-2 therapy showed a relatively higher response rate and toxicities, while a low-dose continuous and subcutaneous regimen resulted in a relatively lower response rate and toxicities. ${ }^{18,22}$ On the other hand, because many HD IL-2 studies have not shown survival benefits compared to those associated with alternative regimen studies, low-dose continuous and subcutaneous regimens are still used in clinical practice. Therefore, the choice of the therapeutic IL-2 regimen must be based on the patient's conditions.

Many studies have shown differences in the natural history of metastatic RCC between racial and ethnic groups. ${ }^{23,24}$ Many studies have already shown significant differences in AEs between Asian and non-Asian populations. ${ }^{25}$ However, there have not been enough studies on the side effects of IL-2 according to race. Lee et al. ${ }^{3}$ demonstrated the necessity of modifying the HDIL-2 treatment schedule in Asian patients because of severe AEs. The most common AEs of grade 3 or higher were anuria (84\%), hypotension (81\%), and thrombocytopenia (81\%). They suggested that a long rest between treatment cycles is needed for Asian patients. However, in this study, there were no life-threatening treatment-related AEs, such as hypotension and sepsis. One patient complained of grade 4 general weakness but fully recovered after supportive care. Most toxicities were mild to moderate, and no patient required a dose reduction owing to AEs. Although this study population was highly vulnerable to AEs, the incidence of serious AEs was low. This result may be attributable to the safety of the subcutaneous regimen.

Cytokines inhibit tumor cell growth directly by antiproliferative or proapoptotic activity or indirectly by stimulating cytotoxic immune cells against the tumor. There had been high expectations of cytokine antitumor activity after the discovery of cytokines. However, studies on cytokines failed to show meaningful outcomes. IL-2 had especially been receiving a lot of attention because it is a key molecule in the activation of NK cells and cytotoxic T-lymphocytes. ${ }^{26}$ However, it demonstrated only mild clinical benefit and a high level of toxicities. These limitations are considered to originate from the short half-life of most cytokines and the complexities of cell signal transduction. Therefore, there were several difficulties in using IL-2 in clinical practice. For more than a decade, TKIs have been commonly used to treat metastatic RCC, after the advent of new antiangiogenic agents. In recent times, ICIs have come to represent a revolution in cancer treatment, and immunotherapy is currently receiving a lot of attention. Cytokines are being studied in clinical trials, mainly in conjunction with anti-PD-1 and anti-PD-L1 monoclonal antibodies. Basically, tumor antigens are presented by antigen-presenting cells followed by modulation of $\mathrm{T}$-cell activity by immune checkpoints on the cell membrane of $\mathrm{T}$ cells, the blockade or stimulation of which consequently results in increased T-cell activity, a key step in increasing the antitumor immune response. Therefore, IL-2 is still important for effective cancer therapy, and several studies on nextgeneration IL-2, with improved pharmacokinetics and pharmacodynamics, are underway. ${ }^{27,28}$

This study has some limitations. First, this study was a prospective, single-arm, noncomparative study was aimed at assessing the objective tumor response rate, and although the target sample of 21 participants was met, the sample was still small to ensure generalizability of the findings. Therefore, caution is needed when generalizing the study results. Second, this study aimed to observe the responses following drug administration in clinical practice and 
not to compare the therapeutic effects with those of other agents. Thus, it is important to note that the therapeutic effects of the study treatment cannot be objectively compared to those of other drugs. Third, this study has some unmeasured confounding factors and potential biases that could account for the observed associations. To reduce potential confoundings, the overall response was assessed independently by researchers at each facility and another independent tumor assessor, and their agreement was examined. In the final analysis, the results of the assessment by the researchers at each facility were included. Fourth, we could not present data on overall survival and cancer-specific survival, which limits the acceptance of our findings.

\section{CONCLUSIONS}

A subcutaneous IL-2 regimen might be a safe and effective treatment with respect to tumor response and survival in Korean patients with metastatic RCC. Although it is difficult to use this treatment as the first-line treatment option for metastatic RCC in the target therapy era, it is still worth considering for select patients with metastatic RCC.

\section{CONFLICT OF INTEREST}

This work was supported by BMIKOREA, Inc., Jeju, South Korea. Except for that, the authors claim no conflicts of interest.

\section{ORCID}

Jeong Ho Kim, https://orcid.org/0000-0003-4491-0308

Ki Soo Lee, https://orcid.org/0000-0003-0825-3996

Choung-Soo Kim, https://orcid.org/0000-0002-7464-3207

Young Deuk Choi, https://orcid.org/0000-0002-8545-5797

Tae Hyo Kim, https://orcid.org/0000-0002-5994-7878

\section{REFERENCES}

1. Gupta K, Miller JD, Li JZ, Russell MW, Charbonneau C. Epidemiologic and socioeconomic burden of metastatic renal ceel carcinoma (mRCC): a lterature review. Cancer Treat Rev 2008;34:193-205.

2. Heng DY, Xie W, Regan MM, Harchman LC, Bjarnason GA, Vaishampayan UN, et al. External validation and comparison with other models of the International Metastatic Renal-Cell Carcinoma Database Consortium prognostic model: a population-based study. Lancet Oncol 2013;14:141-8.

3. Lee JY, Song C, Hong BS, Hong JH, Ahn H, Lee JL. Clinical outcome of high-dose bolus intravenous interleukin-2 with a modified administration schedule for Asian patients with metastatic renal cell carcinoma. Cancer Chemother Pharmacol 2017;79:173-80.

4. McDermott DF, Cheng SC, Signoretti S, Margolin KA, Clark JI, Sosman JA, et al. The high-dose aldesleukin "select" trial: a trial to prospectively validate predictive models of response to treatment in patients with metastatic renal cell carcinoma. Clin Cancer Res 2015;21:561-8.

5. Motzer RJ, Hutson TE, Cella D, Reeves J, Hawkins R, Guo J, et al. Pazopanib versus sunitinib in metastatic renal-cell carcinoma. N Engl J Med 2013;369: 722-31.

6. Motzer RJ, Hutson TE, Tomczak P, Michaelson MD, Bukowski RM, Rixe O, et al. Sunitinib versus interferon alfa in metastatic renal-cell carcinoma. N Engl J Med 2007;356:115-24.

7. Motzer RJ, Escudier B, McDermott DF, George S, Hammers HJ, Srinivas S, et al. Nivolumab versus everolimus in advanced renal-cell carcinoma. $\mathrm{N}$ Engl J Med 2015;373:1803-13.

8. Motzer RJ, Rini BI, McDermott DF, Frontera OA, Hammers HJ, Carducci MA, et al. Nivolumab plus ipilimumab versus sunitinib in first-line treatment for advanced renal cell carcinoma: extended follow-up of efficacy and safety results from a 
randomised, controlled, phase 3 trial. Lancet Oncol 2019;20:1370-85.

9. Diamond E, Molina AM, Carbonaro M, Akhtar NH, Giannakakou P, Tagawa ST, et al. Cytotoxic chemotherapy in the treatment of advanced renal cell carcinoma in the era of targeted therapy. Crit Rev Oncol Hematol 2015;96:518-26.

10. Dutcher JP, Nanus D. Long-term survival of patients with sarcomatoid renal cell cancer treated with chemotherapy. Med Oncol 2011;28:1530-3.

11. Nanus DM, Garino A, Milowsky MI, Larkin M, Dutcher JP. Active chemotherapy for sarcomatoid and rapidly progressing renal cell carcinoma. Cancer 2004;101:1545-51.

12. Oudard S, Banu E, Vieillefond A, Fournier L, Priou F, Medioni J, et al. Prospective multicenter phase II study of gemcitabine plus platinum salt for metastatic collecting duct carcinoma: results of a GETUG (Groupe d'Etudes des Tumeurs Uro-Genitales) study. J Urol 2007;177:1698-702.

13. Chang AJ, Zhao L, Zhu Z, Boulanger K, Xiao H, Wakefield MR, et al. The past, present and future of immunotherapy for metastatic renal cell carcinoma. Anticancer Res 2019;39:2683-7.

14. Berraondo P, Sanmamed MF, Ochoa MC, Etxeberria I, Aznar MA, Pérez-Graciet JL, et al. Cytokines in clinical cancer immunotherapy. Br J Cancer 2019;120:6-15.

15. Bukowski RM. Natural history and therapy of metastatic renal cell carcinoma: the role of interleukin-2. Cancer 1997;80:1198-220.

16. Fyfe G, Fisher RI, Rosenberg SA, Sznol M, Parkinson DR, Louie AC. Results of treatment of 255 patients with metastatic renal cell carcinoma who received high-dose recombinant interleukin-2 therapy. J Clin Oncol 1995;13:688-96.

17. Buter J, Sleijfer DT, van der Graaf WT, de Vries EG, Willemse PH, Mulder NH. A progress report on the outpatient treatment of patients with advanced renal cell carcinoma using subcutaneous recombinant interleukin-2. Semin Oncol 1993;20(6 Suppl
9):16-21.

18. Yang JC, Sherry RM, Steinberg SM, Topalian SL, Schwartzentruber DJ, Hwu P. Randomized study of high-dose and low-dose interleukin-2 in patients with metastatic renal cancer. J Clin Oncol 2003;21: 3127-32.

19. Sleiifer DT, Janssen RA, Buter J, de Vries EG, Willemse $\mathrm{PH}$, Mulder NH. Phase II study of subcutaneous interleukin-2 in unselected patients with advanced renal cell cancer on an outpatient basis. J Clin Oncol 1992;10:1119-23.

20. Lara PN Jr, Redman MW, Kelly K, Edelman MJ, Williamson SK, Crowley JJ, et al. Disease control rate at 8 weeks predicts clinical benefit in advanced non-small-cell lung cancer: results from Southwest Oncology Group randomized trials. J Clin Oncol 2008;26:463-7.

21. Fisher RI, Rosenberg SA, Fyfe G. Long-term survival update for high-dose recombinant interleukin-2 in patients with renal cell carcinoma. Cancer J Sci Am 2000;6 Suppl 1:S55-7.

22. Yang JC, Topalian SL, Parkinson D, Schwartzentruber DJ, Weber JS, Ettinghausen SE, et al. Randomized comparison of high-dose and low-dose intravenous interleukin-2 for the therapy of metastatic renal cell carcinoma: an interim report. J Clin Oncol 1994;12:1572-6.

23. Hoffmann SC, Stanley EM, Cox DE, DiMercurio BS, Koziol DE, Harlan DM, et al. Ethnicity greatly influences cytokine gene polymorphism distribution. Am J Transplant 2002;2:560-7.

24. Naito S, Yamamoto N, Takayama T, Muramoto M, Shinohara N, Nishiyama K, et al. Prognosis of Japanese metastatic renal cell carcinoma patients in the cytokine era: a cooperative group report of 1463 patients. Eur Urol 2010;57:317-25.

25. Guo J, Jin J, Oya M, Uemura H, Takahashi S, Tatsugami K, et al. Safety of pazopanib and sunitinib in treatment-naive patients with metastatic renal cell carcinoma: Asian versus non-Asian subgroup analysis of the COMPARZ trial. J Hematol Oncol 2018; 
11:69.

26. Motzer RJ, Bander NH, Nanus DM. Renal-cell carcinoma. N Engl J Med 1996;335:865-75.

27. Skrombolas D, Frelinger JG. Challenges and developing solutions for increasing the benefits of IL-2 treatment in tumor therapy. Expert Rev Clin Im- munol 2014;10:207-17.

28. Sun Z, Ren Z, Yang K, Liu Z, Cao S, Deng S, et al. A next-generation tumor-targeting IL-2 preferentially promotes tumor-infiltrating CD8(+) T-cell response and effective tumor control. Nat Commun 2019;10:3874. 